\title{
Vom Wiener Stadtphysikat zum Gesundheitsamt der Stadt Wien ${ }^{1}$
}

\author{
From the Viennese Stadtphysikat to the Public Health Department of Vienna
}

\section{Bibliografie}

Dol http://dx.doi.org/

10.1055/s-0033-1344193

Online-Publikation: 11.7.2013

Pneumologie 2013; 67: 448-453

(c) Georg Thieme Verlag KG

Stuttgart · New York

ISSN 0934-8387

Korrespondenzadresse Hofrat Dr. Ermar Junker Landessanitätsdirektor i. R. Vorgartenstraße 158/1/3 1020 Wien

Austria

dr.ermar.junker@hotmail.com
Im Jahre 1540 beschloss die Wiener Medizinische Fakultät, die Stelle eines eigenen Sanitätsbeamten für die Stadt Wien zu schaffen. Die Regierung war bereit, die Besoldung dieses Arztes zu übernehmen und gab ihm den Namen „Magister Sanitatis“. Mit diesem Amte sollte stets nur ein Mitglied der Fakultät betraut werden.

Die Errichtung eines Magisterium Sanitatis für die Stadt war eine Notlösung. Die Fakultät hatte damals sehr viele Aufgaben zu erfüllen. Sie musste die Studenten ausbilden, hatte in der Funktion einer Standesorganisation mit Zunftcharakter die Interessen der in Wien niedergelassenen freipraktizierenden Ärzte zu vertreten und musste als sanitäre Aufsichtsbehörde die Agenden des öffentlichen Gesundheitswesens wahrnehmen. Gerade der letztgenannten Verpflichtung konnte sie nur schwer nachkommen. Viele unangenehme Aufgaben waren damit vor allem in Epidemiezeiten verbunden. Die Regierung und die Stadtverwaltung traten oft an sie um Rat und Unterstützung heran. Die Abstellung von Ärzten in Notzeiten war für die Fakultät besonders belastend. Außerdem war sie schon im Jahre 1501 verpflichtet worden, Arme unentgeltlich zu behandeln, und im Jahre 1517 gab ihr Kaiser Maximilian I. den Auftrag, der Stadt Wien je einen Armen- und Spitalsarzt unentgeltlich zur Verfügung zu stellen. Die sanitären Aufgaben konnte man nun, zumindest teilweise, dem Magister Sanitatis übertragen, und Regierung und Stadtverwaltung mussten nun nicht mehr in Epidemiezeiten die Fakultät um Überlassung eines Infektionsarztes bitten, der gerade in diesen Zeiten nicht leicht zu bekommen war.

\footnotetext{
${ }^{1}$ Erstveröffentlichung in Wiener Medizinische Wochenschrift 1975; 125: 18-23. Nachdruck mit freundlicher Genehmigung der Springer-Verlag GmbH.
}

Auch in anderen deutschen Städten waren durch die Seuchenplage die Stadtverwaltungen gezwungen, eigene Stadtärzte anzustellen, die als Stadtphysici ${ }^{2}$ bezeichnet wurden.

In Wien entschloss sich die Regierung zu diesem Schritt, und die verheerenden Pestepidemien waren der Anlass, das Magisterium Sanitatis für die Stadt zu schaffen.

Doch dieser Magister Sanitatis hatte keine angenehme Beschäftigung. Er hatte lediglich als fixbesoldeter Arzt die Aufgabe, die Pestkranken zu behandeln; eine Privatpraxis war ihm verboten. Als Beamter des Kaisers war sein Sold sehr niedrig, und daher ist es nicht verwunderlich, dass sich zunächst kein Arzt fand, das Magisterium Sanitatis zu übernehmen. Um aus dieser Zwangssituation herauszukommen, sollte nun über höchsten Auftrag das jüngste Mitglied der Fakultät mit diesem Amte betraut werden. Dieses hatte aber keine Lust, als Pestarzt zu sterben, und lehnte ab. Nun erklärte sich Dr. Franz Vesalius, ein Bruder des großen Anatomen Andreas Vesalius (15151564), bereit, diesen Posten zu übernehmen. Obwohl er kein Mitglied der Fakultät war, wurde er von ihr zum ersten Magister Sanitatis Wiens vorgeschlagen. Bald raffte ihn die Pest dahin. Seinen Nachfolger ereilte das gleiche Schicksal, und im Jahre 1558 sah sich die Fakultät neuerlich gezwungen, ihr jüngstes Mitglied, Dr. Johann Aicholtz, zum Magister Sanitatis vorzuschlagen. Mit wichtigeren Aufgaben betraut, konnte dieser einen Ersatzmann, Dr. Andreas Dadius, nennen, dem, als er sich bei drohender Gefahr zeitgerecht zurückziehen wollte, die Fakultät erklärte, dass jedem einzelnen Fakultätsmitglied sein Leben ge-

\footnotetext{
2 Physikus: Ursprünglich die Bezeichnung für Arzt im Gegensatz zum Chirurgen, später allgemein für Leibarzt oder öffentlichen Arzt gebraucht; Stadtphysikus für Stadtarzt; Landphysikus für Arzte der Länder als Kreisarzt oder Viertelarzt, auch Physiker bezeichnet. Physikat: Amt eines Physikus, Gesundheitsamt.
} 
nauso teuer sei und dass es nur billig wäre, wenn Dadius, der sein Amt in guten Zeiten führte, dieses jetzt auch bei drohender Gefahr behalte (Senfelder). Trotzdem musste auf Befehl der Regierung die Fakultät einige Ärzte für das Magisterium Sanitatis namhaft machen.

Da sich kein geeigneter Arzt finden ließ, wurde ein Student der Medizin, Johann Neumann, im Jahre 1561 vorgeschlagen, der auch das Dekret als Magister Sanitatis erhielt. Bald passte der Regierung diese Regelung nicht, und es wurde neben dem Studenten der aus Italien zugezogene Arzt Dr. Jakob Hartl mit ins Magisterium genommen. Für beide bedeuteten die Ernennungsdekrete ein Fahrbillett ins ]enseits, denn Hartl starb kurz darauf an Pest, während Neumann noch Zeit fand den Doktortitel zu erwerben, um 1568 der Seuche zum Opfer zufallen (Senfelder).

Nun musste die Fakultät neuerlich einen Arzt für das Magisterium suchen, fand keinen und schlug einen alten Studenten mit 30 Semestern, Johann Fraesel, der Regierung vor. Doch diesmal lehnte sie ab. Nun griff man wieder auf Dr. Andreas Dadius zurück. Dieser blieb 4 Jahre lang Magister Sanitatis, erkrankte selbst an Pest und verlor 3 Kinder durch diese Seuche.

Im Jahre 1574 forderte eine kaiserliche Resolution, dass stets der jüngste Arzt des Kollegiums ein Jahr lang das Magisterium zu versehen habe, demselben entweder der nächstältere folge, oderwenn inzwischen ein anderer aufgenommen worden ist-dieser an die Reihe komme. Auf Bitten der Fakultät nahm aber der Kaiser diese Resolution zurück, verlangte jedoch, dass auch in $\mathrm{Zu}$ kunft die Fakultät eine geeignete Person vorzuschlagen habe (Senfelder).

In der Zeit von 1574 bis 1653 übte eine Reihe von Ärzten das Amt des Magisters Sanitatis mit mehr oder weniger Glück bei schlechtester Bezahlung aus. Ganz traurig verlief das Schicksal des 1653 zum Magister Sanitatis ernannten Dr. Christoph Plöchinger, über den in den Akten der Fakultät aus dem Jahre 1664 verzeichnet ist, dass er dem Magisterium Sanitatis in löblicher Weise vorstand, mit dem versprochenen Gehalt im Stiche gelassen wurde und mit Hinterlassung von Waisen selig im Herrn entschlafen ist. Er ist den Hungertod gestorben. Plöchingers Geschick war jedenfalls das härteste, welches je einen Wiener Sanitätsbeamten getroffen hatte. So ist es auch verständlich, dass ein anderer Medizinalbeamter, der ständische Viertelarzt von Stockerau, Dr. Georg Loga, dem dann diese Stelle angeboten wurde, mit der Erklärung ablehnte, er wolle nicht an Hunger sterben (Senfelder).

Wie schlecht es damals um die Besoldung des Magister Sanitatis bestellt war, zeigt der Umstand, dass Ferdinand II. im Jahre 1622 der Wiener Judenschaft gestattete, ein Haus für eine Synagoge unter der Bedingung zu kaufen, dass sie alljährlich zur Erhaltung eines Magister Sanitatis beitrage. Dies dürfte auch nicht sehr zur Besserung des Einkommens dieser Medizinalbeamten beigetragen haben, denn die Fakultät musste auch weiterhin sehr darum kämpfen, eine anständige Besoldung für den Magister Sanitatis zu erreichen, damit ein guter Arzt diese Stelle übernehme. Ein Pestarzt musste vorhanden sein, denn obwohl seit 1561 ein Verbot für die Ärzte Wiens bestand, in Seuchenzeiten die Stadt zu verlassen, konnten diese zur Behandlung von Pestkranken nicht gezwungen werden. Später wurde ihnen sogar vorübergehend die Behandlung von Pestkranken verboten. Erst nach der letzten Pestepidemie im Jahre 1713 fanden sich genügend Bewerber um diesen Posten, und zuletzt regelte Maria Theresia zufriedenstellend die leidliche Bezahlungsfrage.

Um 1646 arbeitete die Fakultät eine Instruktion für das Magisterium Sanitatis aus. Diese verpflichtete den Magister Sanitatis, den Verkehr mit Gesunden zu meiden, die Kirche nur in den frühen
Morgenstunden zu besuchen, jeden Pestkranken, ob arm oder reich, zu behandeln, diesen einmal täglich zu besuchen, jeden Pestkranken anzuzeigen, zur Pestzeit nachts in seiner Wohnung zu bleiben, jeden Pestkranken zu trösten und ihn zum baldigen Empfang der Sterbesakramente zu ermahnen. Weiters hatte er den Chirurgus Sanitatis (Pestilenzarzt) und die Totenbeschauer zu überwachen, eine Überbeschau durchzuführen, die Rezepte für arme Pestkranke auf ihre Preiswürdigkeit zu überprüfen und Verdachtsfälle zu untersuchen.

Zur großen Pestzeit im Jahre 1679 amtierte Dr. Christoph Resch als Magister Sanitatis. Ihm musste ein zweiter Arzt in gleicher Stellung beigegeben werden. Beide wurden noch von 4 weiteren Ärzten unterstützt. Damals durfte kein Wiener Arzt die Behandlung Pestkranker ablehnen. Das Collegium oder Consilium Sanitatis, das sich im Laufe der Zeit zu einer Art zentraler Gesundheitsbehörde entwickelte, hatte damals schon auf die Bekämpfung der Seuche einen maßgeblichen Einfluss.

Während der letzten großen Wiener Pestepidemie im Jahre 1713 wurden die beiden Magistri Sanitatis Dr. Johann Georg Schultz und Dr. Christoph Ruck in den Kompetenzstreit zwischen dem mächtig gewordenen Consilium Sanitatis und der Fakultät hineingezogen. Durch den Streit dieser beiden Institutionen wurden wichtige Maßnahmen gegen das Eindringen und die Weiterverbreitung der Pest unterlassen, und die Seuche fand unter dem Volke und den Magistri Sanitatis reiche Ernte. Der Mangel an Ärzten für das Magisterium Sanitatis veranlasste nun die Regierung zu verlangen, die Fakultät soll die Magistri einfach auslosen (Senfelder).

Das Consilium Sanitatis, das unter anderem Namen bereits 1585 in Urkunden erwähnt wird (Slezak), hatte im 18.Jahrhundert bereits einen sehr großen Einfluss im sanitären Bereich und war bestrebt, die Autonomie der Fakultät auf diesem Sektor auszuschalten und diese Belange unter staatliche Kontrolle zu bringen. Da die Fakultät keinerlei Organe hatte, die in der Lage waren, Verordnungen und Verfügungen durchzusetzen, mussten ihre seuchenhygienischen Bemühungen scheitern. Sogar bei der Kontrolle der Sanitätspersonen versagte sie, und in ihrer Not stellte die Fakultät mehrmals an den Passauer Bischof den Antrag, unbotmäßige Quacksalber zu exkommunizieren (Lesky). Umso mehr musste die Fakultät bei der praktischen Durchführung ihrer sanitären Aufgaben versagen. Erst van Swieten als Protomedicus und Präses der Wiener Medizinischen Fakultät konnte hier Ordnung schaffen und die Kompetenzen abgrenzen.

Die immer wieder vom Osten nach Österreich hereinbrechenden Pestepidemien zwangen die Regierung, eigene Pestbehörden zu schaffen. Daraus entstand im Laufe der Zeit eine Körperschaft, für die sich der Titel „consilium sanitatis“ einbürgerte und die später als „commissio sanitatis aulica“ bezeichnet wurde. Dieser zunächst niederösterreichischen Landesbehörde fielen immer weitere Aufgaben zu, durch die diese Körperschaft in die Funktion einer Verwaltung und Hofbehörde aufstieg, aus der sich dann die Sanitäts-Hofdeputation entwickelte (Lesky).

Neben dem Consilium Sanitatis und der Fakultät gab es seit dem Jahre 1577 einen sich ebenfalls immer stärker entwickelnden ständischen Sanitätsapparat. In diesem sogenannten landschaftlichen Sanitätswesen waren 4 Physiker als sogenannte Viertelärzte in jedem Kreis eingesetzt, die ebenfalls von der Fakultät namhaft gemacht werden mussten. Zwischen diesem landschaftlichen Protomedikat und dem Consilium Sanitatis, der landesfürstlichen Gesundheitsbehörde, kam es ebenfalls häufig zu Streitigkeiten und Reibereien, da sich Letztere zur Durchführung 
der von ihr angeordneten Maßnahmen des landschaftlichen Sanitätsapparates bedienen musste.

Dagegen unterstand der Wiener Sanitätsbezirk niemals der landschaftlichen Organisation bzw. der Niederösterreichischen Sanitätskommission, sondern der Regierung und Kammer, dem Consilium Sanitatis und später der Sanitäts-Hofdeputation. Wien besaß also bis ins 19. Jahrhundert hinein keinen eigentlichen Stadtarzt wie andere Städte, sondern einen besoldeten Physikus, der ein Regierungsbeamter war.

Eine Einheit in der Medizinalverwaltung kam erst durch das Sanitäts-Hauptnormativ aus dem Jahre 1770 zustande. In diesem wichtigen Sanitätsgesetz sind die Sanitäts-Hofdeputation als Zentralbehörde und die Sanitätskommissionen als oberste Landesbehörde bei den Landesregierungen verankert. Im Jahre 1773 wurde nachträglich für jedes Land ein Landes-Protomediker mit dem Titel „Sanitätsrat“ vorgeschrieben. Der Wirkungsbereich des Landesprotomedikers wurde 1806 neu festgelegt. Er umfasste die Überwachung des allgemeinen Gesundheitszustandes der Bevölkerung, die Aufsicht über die Sanitätspersonen, die Überwachung der Totenbeschau, die periodische Untersuchung der Apotheken, die Kontrolle der Heilbäder und Gesundbrunnen, die ärztliche Aufsicht über die Kranken-, Humanitäts- und Strafanstalten, die Handhabung der bei Epidemien und Tierseuchen notwendigen Maßregeln und die Vorlage von Sanitätsberichten. In der zweiten Hälfte des vorigen Jahrhunderts erhielt der Protomediker die Bezeichnung „Landesmedizinalrat“, und die nebenberufliche Praxisausübung war ihm gestattet (Slezak).

Im Laufe des 18. und 19. Jahrhunderts erweiterten sich langsam die Tätigkeitsbereiche der Magistri Sanitatis und näherten sich allmählich dem Wirkungskreis der heutigen Amtsärzte. Sie fungieren nicht nur mehr allein als Epidemieärzte, sondern haben auch viele allgemeine sanitätspolizeiliche Aufgaben durchzuführen. Ab 1782 wird für Magister Sanitatis auch die Bezeichnung Sanitätsmagister gebraucht.

Im Jahre 1795 wird Dr. Nikolaus v. Paraskovitz zum ersten Stadtphysikus und Dr. Andreas Stifft zum zweiten Stadtphysikus und Sanitätsmagister ernannt. Sie unterstanden der Niederösterreichischen Landesregierung, der Polizeidirektion, dem Stadtmagistrat und dem Landesgericht (Senfelder).

In den Sanitätsberichten scheinen immer wieder sogenannte Armenärzte auf. In Wien gab es bis zum Jahre 1784 den „Armenleut-Doktor“, der ursprünglich am Rennweg, später im spanischen Spital den Armen aus allen Bezirken Wiens unentgeltlich ärztlichen Rat erteilte und Arzneien verabreichte. Von 1786 an besorgten zwei eigens dazu angestellte Spitalsärzte bei einer Frühordination im Allgemeinen Krankenhaus dieses Geschäft. Schwerkranke Patienten konnten sogleich im Spital aufgenommen werden. Da diese armenärztliche Betreuung aus verschiedenen Gründen nicht funktionierte und für viele Kranke der Anmarschweg zu weit war, entschloss sich Kaiser Leopold II. im Jahre 1791, zusätzlich noch abgesonderte Polizeibezirkskrankenanstalten einzuführen und zu organisieren. Im Jahre 1796 schrieb dann Kaiser Franz I. mit einem Hofbescheid den PolizeibezirksÄrzten, den Polizeibezirks-Wundärzten und den PolizeibezirksHebammen eine Dienstinstruktion vor, durch die sie auch verpflichtet waren, Hausbesuche durchzuführen. Die Armenordination im Allgemeinen Krankenhaus wurde aufgelöst und in jedem Polizeibezirk die Stelle eines Bezirksarztes, eines Bezirkswundarztes und einer Hebamme systemisiert. Die Besetzung dieser Ämter erfolgte durch die k. k. Polizei- und Censur-Hofstelle. Für die Innere Stadt wurde die Stelle eines Stadtarmenarztes geschaffen. Seine Pflichten umfassten teils medizinische und teils wis- senschaftliche Gegenstände. Was letztere betrifft, hatte er merkwürdige Krankheiten zu beschreiben, neue Heilmethoden zu prüfen und belehrende Leichenöffnungen niemals zu unterlassen. Er musste sich ebenso wie die Bezirkspolizeiärzte an jedem 10. Werktage in jedem Monat nachmittags um $4 \mathrm{Uhr}$ in der Wohnung des ersten Stadtphysikus zur Besprechung einfinden (Knolz). Mit Erlass der k. k. niederösterreichischen Stadthalterei vom 2. Jänner 1867 wurde dann jeder 3. Samstag der Monate zur gemeinsamen Versammlung bestimmt.

Erst im Jahre 1892 wurden die Polizeiärzte vom armenärztlichen Dienst enthoben. Die damals getroffene Neuregelung des Dienstes der Amtsärzte der k. k. Polizeidirektion in Wien gab diesen allgemeine sanitätspolizeiliche Aufgaben, die nach dem Reichssanitätsgesetz 1870 die kommunalen Behörden allein zu erfüllen hatten. Dadurch entstanden parallele Tätigkeitsbereiche. Die städtischen Ärzte protestierten energisch gegen diese Regelung. Die gemeinsamen Sitzungen wurden abgesagt, und der Polizeichefarzt nahm nur noch als Delegierter an den unter dem Vorsitz des Stadtphysikus allmonatlich stattfindenden amtsärztlichen Versammlungen teil.

Im Jahre 1857 verfügte Wien bereits über 2 Stadtphysiker, einen Stadtwundarzt, 16 Armenärzte, 4 Armenwundärzte, 3 Armenaugenärzte und 4 Totenbeschauer. Es war dies nur ein bescheidener Anfang; der Wiener Sanitätsdienst war zu dieser Zeit noch sehr zersplittert und uneinheitlich aufgebaut. Daher hatte auch bis zum Jahre 1864 das Stadtphysikat von Wien kaum den Anspruch auf diese Bezeichnung machen können, da es kein eigentliches Amt darstellte, sondern nur von 2 Amtsärzten repräsentiert war. Bis zu diesem Jahr hatte Dr. Sigmund Michael Granichstädter das Stadtphysikat inne. Er führte als letzter den offiziellen Titel Sanitätsmagister. In den Jahren 1864 bis 1881 leiteten Dr. Eduard Nusser und Dr. Franz Innhauser diese Stelle. Sie wiesen schon im Jahre 1864 auf die Notwendigkeit hin, alle in Wien im Sanitätsdienst tätigen Organe der Kommune unterzuordnen (Grünberg). Seit ihrem Amtsantritt werden auch regelmäßige Publikationen vom Stadtphysikat veröffentlicht, die einen wertvollen Überblick über den Gesundheitszustand der Bevölkerung und einen guten Einblick in die Entwicklung des Gesundheitswesens in Wien geben.

Erst mit Inkrafttreten des Reichssanitätsgesetzes vom 30. April 1870 wurden die Stadtphysiker städtische Beamte und dem Magistrat der Stadt Wien unterstellt.

Nach geraumer Zeit, in Jahre 1880, wurde eine Instruktion für die städtischen Ärzte herausgegeben, die zunächst lediglich die Totenbeschau regelte, und im Jahre 1881 wurde beschlossen, nur einen Stadtphysikus und 2 Stellvertreter zu ernennen. Dr. Emil Kammerer erhielt die Stelle. Im Jahre 1898 wurde er als erster zum Ober-Stadtphysikus ernannt.

Das Jahr 1892 brachte die Einbeziehung der Vororte in das Wiener Gemeindegebiet. Die Gemeinde übernahm fast alle vorortlichen Amtsärzte und die 34 Armenärzte, die die amtliche Bezeichnung „städtische Arzte für Armenbehandlung und Totenbeschau“ erhielten. Vorübergehend gab es dann in den neuen Bezirken städtische Arzte und in den alten k. k. Armen- und Polizeiärzte. Im Jahre 1895 verfasste der damalige Stadtphysikus Dr. E. Kammerer ein Organisationsstatut, in dem schon fast alle Agenden verankert sind, die auch heute noch die Grundlage der sanitätspolizeilichen und gutachterlichen Tätigkeit des Gesundheitsamtes darstellen. Darin ist festgehalten, dass der Stadtphysikus und seine beiden Stellvertreter die sachverständigen Sanitätsorgane der Gemeinde Wien in sämtlichen, der zentralen Verwaltung vorbehaltenen Angelegenheiten und für jene sanitären Agenden 
der Magistratischen Bezirksämter, welche eine prinzipielle Bedeutung beanspruchen, durch die Besonderheit des Falles hervorragen oder die Interessen mehrerer Bezirke oder des ganzen Stadtgebietes berühren, sind. Die den Magistratischen Bezirksämtern zugewiesenen städtischen Bezirksärzte unterstehen dem Leiter des Magistratischen Bezirksamtes, die dem Stadtphysikat zugewiesenen Bezirksärzte sind unmittelbar dem Stadtphysikus untergeordnet.

Das Reichssanitätsgesetz aus dem Jahre 1870 brachte einen grundlegenden Wandel im Aufbau des Sanitätswesens in Österreich und dessen weiterer Entwicklung. Dieses moderne Sanitätsgesetz beauftragt die politischen Behörden mit der Durchführung der Sanitätsangelegenheiten und legt die instanzenmäßige Gliederung fest, die bis heute beibehalten wurde. Das Gesetz bestimmte auch die Konstituierung der Landessanitätsräte und des Obersten Sanitätsrates als beratende Kollegien. Zum Mitglied des Obersten Sanitätsrates für die erste Funktionsperiode 1870 bis 1873 wurde auch der damalige Stadtphysikus von Wien, Dr. E. Nusser, ernannt.

Ende des Jahres 1910 versahen in den 21 Wiener Gemeindebezirken 32 Bezirksärzte und 99 städtische Ärzte, zusammen also 131 Amtsärzte, und in der Zentrale des Stadtphysikates 7 Amtsärzte den Dienst. Dazu müssen noch die Ärzte des augen- und ohrenärztlichen Dienstes gerechnet werden. Somit waren in diesem Jahr 144 Amtsärzte im Dienste des Stadtphysikats. Innerhalb von 50 Jahren war ein beachtlicher Sanitätsapparat aufgebaut worden.

Der Verfasser des Organisationsstatuts für das Stadtphysikat, Oberstadtphysikus Dr. E. Kammerer, starb im Jahre 1901. Im Jahre 1902 trat Dr. Theodor Szongott dieses Amt an. Ihm folgte im Jahre 1911 Dr. August Böhm. Er war bereits seit 1909 Mitglied des Obersten Sanitätsrates.

Um die für seuchenhygienische Maßnahmen notwendigen Untersuchungen durchführen zu können, wurde im Jahre 1908 im Hygiene-Institut der Universität Wien eine städtische Untersuchungsstelle geschaffen. Diese wurde dann im Jahre 1925 ins städtische Karolinen-Kinderspital verlegt und bekam erst im Jahre 1951 ein eigenes Haus, das ehemalige Maria-Theresien-Frauenhospiz im 8. Wiener Gemeindebezirk, als Institutsgebäude zugewiesen. Diese Hygienisch-bakteriologische Untersuchungsanstalt wurde zu einem wichtigen Bestandteil des Gesundheitsamtes der Stadt Wien, an dem sehr namhafte Fachleute wirkten.

In den Jahren 1914 bis 1918 bestimmte die Kriegssituation die Arbeit des Stadtphysikates. So wurde im Jahre 1916 auch eine „Städtische Beratungsstelle für Ernährung der Kranken während des Krieges“ innerhalb dieses Sanitätsapparates geschaffen.

Das Ende des 1. Weltkrieges brachte eine grundlegende Änderung des Aufbaues der städtischen Sanitätsbehörden.

Im Laufe des Jahres 1918 wurde das Stadtphysikat in ein städtisches Gesundheitsamt als selbständige Abteilung des Magistrates umgestaltet. Weiters wurde mit dem Erlass der Magistratsdirektion vom 31. Mai 1920 verfügt, dass die Leitung und Oberaufsicht über den gesamten ärztlichen Dienst der Gemeinde bezüglich der Verhütung und Behandlung von Krankheiten und in Angelegenheit der Sozialhygiene der Oberstadtphysikus innehat.

Die Übernahme der Geschäfte einer politischen Landesbehörde durch den Magistrat der Stadt Wien ergab auch im Bereich des Gesundheitsamtes eine wesentliche Erweiterung seines Wirkungsbereiches. Auf Grund des Stadtsenatsbeschlusses vom 19. November 1920 und durch Verfügung des Bürgermeisters vom 27. November 1920 wurde dem Gesundheitsamt der Stadt Wien die Behandlung aller rein fachlichen, vom Wiener Magis- trat als politische Landesbehörde zu besorgenden Sanitätsangelegenheiten (Geschäftsagenden des Landessanitätsreferenten) zugewiesen.

Das Gesundheitsamt wurde zunächst in die Verwaltungsgruppe IV (Sozialpolitik und Gesundheitswesen) eingegliedert und im Jahre 1920 der Gruppe III (Fürsorge) als Magistratsabteilung 12 zugeteilt.

Während sich die Tätigkeit des Stadtphysikats in der 2. Hälfte des vorigen Jahrhunderts vorwiegend auf die Bekämpfung der Seuchen und auf die Durchführung allgemeiner sanitärer Maßnahmen erstreckte, wobei es wichtige Beraterfunktionen auf sanitärem Gebiet bei der Entwicklung Wiens zur Großstadt innehatte, war der Arbeitsbereich und die Fortentwicklung des Gesundheitsamtes nach dem 1.Weltkrieg vorwiegend auf gesundheitsfürsorgerische und sozialmedizinische Bereiche ausgerichtet.

Zunächst galt es aber, die letzte große Seuche, die Tuberkulose, unter Kontrolle zu bringen und den Tuberkulosekranken zu helfen. Im Jahre 1919 wurde die erste städtische Tuberkulosefürsorgestelle gegründet und im Jahre 1921 die „Landeszentrale zur Bekämpfung der Tuberkulose“ geschaffen, deren Agenden vom Oberstadtphysikus als Landessanitätsreferenten wahrgenommen wurden, dem ein ärztlicher Vollzugsausschuss zur Seite stand. In das Jahr 1923 fiel die Gründung einer Zentralaufnahmestelle für Tuberkulöse und Kurbedürftige beim städtischen Gesundheitsamt, der die Errichtung einer eigenen Sputumsuntersuchungsstelle im Jahre 1927 und einer städtischen Stelle zur Prüfung der Sinkgeschwindigkeit des Blutes (Fahraeus-Station) im Jahre 1929 folgten. Chefarzt Doz. Dr. A. Götzl war bis zum Jahre 1938 der Leiter dieser ausgezeichneten Einrichtung der Tuberkulosebekämpfung. Erst nach 1938 wurde die Tuberkulosefürsorgearbeit den staatlichen Gesundheitsämtern als Pflichtaufgabe übertragen, zu der die Dritte Durchführungsverordnung zum Gesetz über die Vereinheitlichung des Gesundheitswesens als deutsche Rechtsvorschrift die Grundlage bildete.

Als weitere wichtige gesundheitsfürsorgerische Maßnahme wurde im Jahre 1919 der erweiterte schulärztliche Dienst für alle Volks- und Bürgerschulen eingeführt und die Errichtung von städtischen Schulzahnkliniken beschlossen. Die erste Schulzahnklinik öffnete im Jahre 1922 ihre Pforten. Drei Jahre später erfolgte die Errichtung der „gesundheitlichen Beratungsstelle für Ehewerber" und die Eingliederung des Referates für ärztliche Belange der Jugendfürsorge in das Gesundheitsamt. Bereits in den Jahren 1923 und 1924 konnten städtische Abendambulatorien zur Behandlung Geschlechtskranker in Betrieb genommen werden. Im Jahre 1924 wurden auch die „Fürsorgestelle für Trunksüchtige“ beim Gesundheitsamt aufgebaut und 1926 die „Beratungsstelle für Nervenkranke und Gemütskranke“ geschaffen.

Durch die im Jahre 1934 getroffene neue Geschäftseinteilung wurde die Magistratsabteilung 12 als Magistratsabteilung 19 in die Gruppe III (Wohlfahrtsamt, Soziale Fürsorge und Gesundheitswesen) eingegliedert; im Jahre 1937 eine Fürsorgestelle für Körperbehinderte eingerichtet.

Oberstadtphysikus Dr. A. Böhm trat im Jahre 1930 in den Ruhestand, und Dr. Viktor Gegenbauer, der seit 1925 die Leitung der städtischen Untersuchungsstelle innehatte, wurde zum neuen Leiter des Gesundheitsamtes ernannt.

Das Jahr 1938, die Einverleibung ins Deutsche Reich, brachte dem Gesundheitsamt einschneidende Veränderungen, vor allem auf dem personellen Sektor. Von den damals im Gesundheitsamt tätigen 195 Ärzten waren 67 jüdischer Abstammung. Sie wurden aufgrund der Nürnberger Gesetze pensioniert oder gekündigt. In diesem Jahr stand einem Abgang von insgesamt 111 Ärzten ein 
Zugang von 129 nebenamtlichen oder hauptamtlichen Ärzten gegenüber.Eine Abteilung für Erb- und Rassenpflege wurde geschaffen. Im Organisationsplan des Reichsgaues Wien aus dem Jahre 1943 scheint das Hauptgesundheitsamt in der Hauptabteilung E-Gesundheitswesen und Volkspflege-unter E 1 bis E 4 auf. Stadtmedizinaldirektor Dr. Hermann Vellguth aus Hannover löste als Leiter des Hauptgesundheitsamtes Oberstadtphysikus Dr. Gegenbauer ab, der im Jahre 1939 auf tragische Weise aus dem Leben schied.

Nach Kriegsschluss im Jahre 1945 war der Organisationsapparat des Gesundheitsamtes schwer angeschlagen und musste neu aufgebaut werden. Völlig zerstört waren 4 Bezirksgesundheitsämter, 3 Tuberkulosefürsorgestellen, 3 Schulzahnkliniken, die fahrbare Schulzahnklinik, die Beratungsstelle für Geschlechtskranke, 7 Mutterberatungsstellen, die fahrbare Mutterberatung, die Absonderungsanstalt und die Desinfektionsanstalt. Auch das Gebäude des Gesundheitsamtes war durch Bombenangriffe und bei den Kampfhandlungen so arg beschädigt worden, dass der Dienst zunächst dort nicht aufgenommen werden konnte. Die Bezirksgesundheitsämter waren bei ihrer schweren Arbeit oft auf sich allein gestellt. Dennoch konnten die Gesundheitsdienste die damals herrschenden Typhus- und Ruhr-Epidemien rasch unter Kontrolle bringen. Im Jahre 1945 starben in Wien 700 Personen an Bauchtyphus, 113 Personen an Paratyphus und 2926 Personen an Ruhr.

Im Jahre 1946 wurde eine Änderung der Geschäftseinteilung des Magistrates vorgenommen. Das Gesundheitsamt wurde in die Geschäftsgruppe V - Gesundheitswesen und Gesundheitsfürsorge - eingereiht. Unter der Bezeichnung „Magistratsabteilung 15 - Gesundheitsamt“ bestand es aus folgenden Referaten:

- Allgemeine Verwaltungs- und Wirtschaftsangelegenheiten

- Personalbüro und Kanzleileitung

- Berichterstattung, amtsärztliche Ausbildung und Einzelagenden

- Amts- und vertrauensärztliche Untersuchungsstelle

- Hygienereferat

- Seuchenreferat

- Hebammenwesen, sanitäre Übelstände, Leichenwesen

- Sanitätspersonen, Apothekenwesen, Sanitätsgewerbe, Kranken- und Kuranstalten (sanitäre Aufsicht)

- Schulärztlicher Dienst

- Schulzahnkliniken

- Säuglings- und Kleinkinderfürsorge

- Tuberkulosereferat

- Geschlechtskrankenfürsorge

- Fürsorge für Nerven- und Gemütskranke

- Körperbehindertenfürsorge

- Rezeptprüfstelle

- Zusätzliche Krankenernährung

Dem Gesundheitsamt unterstanden damals 26 Bezirksgesundheitsämter, 20 Tuberkulose-Fürsorgestellen, 3 Sputumuntersuchungsstellen, 1 Zentralröntgenstelle, 1 augenärztliche Untersuchungsstelle, 1 Hygienisch-bakteriologische Untersuchungsanstalt, 2 Desinfektionsanstalten, 14 Schulzahnkliniken und 78 Mutterberatungsstellen.

Nach Kriegsschluss ernannte die Stadtverwaltung Dr. Ehrenfried Lande zum Leiter des Gesundheitsamtes. Ihm folgten im Jahre 1960 Dr. Ferdinand Dirschmid und nach dessen Pensionierung im Jahre 1967 Dr. Albert Krassnig als Oberstadtphysikus von Wien. Er wurde im Jahre 1972 als Sektionschef in das neugeschaffene Ministerium für Gesundheit und Umweltschutz berufen.
Der Wiederaufbau der zerstörten Einrichtungen des Gesundheitsamtes konnte schon in den ersten Nachkriegsjahren abgeschlossen werden. Gleichzeitig war es möglich, den in der Zwischenkriegszeit begonnenen und durch politische und kriegsbedingte Umstände unterbrochenen Aufbau einer umfassenden gesundheitsfürsorgerischen Betreuung der Bevölkerung Wiens fortzusetzen. So konnte eine Reihe von neuen Einrichtungen im Gesundheitsamt geschaffen werden: Die BCG-Impfstelle (1948), die Gesundenuntersuchungsstelle (1950), die sportärztliche Untersuchungs- und Beratungsstelle (1950), die Impfstelle für Ausreisende (1955), die ohrenärztliche Untersuchungsstelle (1963), die Alkoholikerberatungstelle (1960), die Narkosestation der Jugendzahnklinik (1965), die Kieferorthopädie der Jugendzahnklinik (1966), in Zusammenarbeit mit der Universitäts-Kinderklinik das Laboratorium für angeborene Stoffwechselstörungen mit Hirnschadensfolgen (1966), die Untersuchungsstelle für Tropenkrankheiten (1966), die Raucherberatungsstelle gemeinsam mit dem Hygiene-Institut der Universität (1973), das Zentrum für Entwicklungsdiagnostik und zerebrale Bewegungsstörungen gemeinsam mit der Universitäts-Kinderklinik (1973) und die Beratungsstelle der Stadt Wien für Eheberatung und Familienplanung gemeinsam mit dem Sozialamt der Stadt Wien (1974). Im Jahre 1959 wurden die Schwangerenberatungsstellen in das Gesundheitsamt eingegliedert. Seit 1974 befindet sich das Gesundheitsamt in der Geschäftsgruppe IV - Soziales und Gesundheit.

Seit mehr als 400 Jahren gibt es in Wien Ärzte, die eigens damit beauftragt sind, die gesundheitlichen Belange der Einwohner dieser Stadt wahrzunehmen. Als Magistri Sanitatis hatten sie zunächst die Funktion eines Pestarztes, und erst im Laufe der Jahrhunderte bekamen sie weitere, allgemeine sanitäre und seuchenhygienische Aufgaben zugewiesen. Vor 100 Jahren wurden diese Beamten der Regierung zu richtigen Stadtärzten, und erst in der 2. Hälfte des vorigen Jahrhunderts war der Aufbau einer wirksamen städtischen Gesundheitsbehörde möglich. Bis dahin verlief die Entwicklung des Stadtphysikats für Wien wesentlich anders als in anderen bedeutenden Städten Europas, die schon viel früher eigene Stadtärzte besaßen. Im Jahre 1918 wurde das Stadtphysikat in ein städtisches Gesundheitsamt umgestaltet. Die Übernahme der Agenden einer Landesbehörde durch den Magistrat der Stadt Wien im Jahre 1920 bedeutete eine wesentliche Aufwertung des Gesundheitsamtes. Seit dieser Zeit ist der Oberstadtphysikus und Leiter des Gesundheitsamtes auch als Landessanitätsreferent der Landessanitätsdirektor von Wien. Das Gesundheitsamt der Stadt Wien zählt heute zu den wichtigsten kommunalen Einrichtungen mit sehr vielseitigen gesetzlichen, gesundheitsfürsorgerischen und sozialmedizinischen Aufgaben.

\section{Literatur}

1 Bericht des Wiener Stadtphysikates über seine Amtsthätigkeit in den Jahren 1891-1893. Wien: 1896

2 Bericht des Wiener Stadtphysikates über seine Amtsthätigkeit in den Jahren 1894-1896. Wien: 1898

3 Bericht des Wiener Stadtphysikates über seine Amtsthätigkeit in den Jahren 1897 bis 1899 . Wien: 1901

4 Bericht des Wiener Stadtphysikates über seine Amtsthätigkeit in den Jahren 1907-1910. Wien: 1913

5 Das neue Wien. Wien: Städtewerk; herausgegeben unter offizieller Mitwirkung der Gemeinde Wien 1927

6 Die Gemeinde-Verwaltung der Reichshaupt- und Residenzstadt Wien in den Jahren 1877-1879. Wien: 1881

7 Die Gemeindeverwaltung der Bundeshauptstadt Wien in der Zeit vom 1. Juli 1919 bis 31. Dezember 1922. Herausgegeben vom Magistrate der Stadt Wien: 1927 
8 Die Gemeinde-Verwaltung der Stadt Wien im Jahr 1938. Verwaltungsbericht Herausgegeben von der Statistischen Abteilung der Gemeindeverwaltung des Reichsgaues Wien: 1941

9 Die Verwaltung der Bundeshauptstadt Wien 1945-1947. Herausgegeben vom Magistrat der Bundeshauptstadt Wien

10 Die Verwaltung der Bundeshauptstadt Wien 1948-1949. Herausgegeben von Magistrat der Bundeshauptstadt Wien

11 Die Verwaltung der Bundeshauptstadt Wien 1950-1951. Herausgegeben vom Magistrat der Bundeshauptstadt Wien

12 Götzl A. Die Tuberkulosefürsorge in Wien, 1919-1929. Wien: 1930

13 Grünberg A. Die Assanierung Wiens vom medizinisch-statistischen Standpunkte.

14 Handbuch der bundesunmittelbaren Stadt Wien. 61. amtlich redigierter Jahrgang
15 Jahres-Bericht des Wiener Stadtphysikates und seine Amtsthätigkeit im Jahre 1882. Wien: 1883

16 Knolz JJ. Darstellung der Humanitäts- und Heilanstalten im Erzherzogthume Österreich unter der Enns. Wien: 1840

17 Krassnig A. Das städtische Gesundheitswesen Wiens der letzten fünfzig Jahre. Hefte 49, 50, 51/52 vom Jahr 1968, 1 und 2 vom Jahr 1969. Stadt Wien

18 Lesky E. Österreichisches Gesundheitswesen im Zeitalter des aufgeklärten Absolutismus. Archiv für österreichische Geschichte

19 Öhler L. Persönliche Mitteilungen.

20 Senfelder L. Geschichte des Wiener Stadtphysikats.

21 Slezak P. Geschichte der österreichischen Sanitätsverwaltung. Wien: Urban \& Schwarzenberg; 1956 